Ann. Sci. forest., 1979, 36 (4), 321-330.

\title{
Comparaison des mesures de surfaces par comptage de points
}

\author{
J. BOUCHON et S. TOMIMURA \\ Station de Sylviculture et de Production, \\ Centre national de Recherches forestières, I.N.R.A., \\ Champenoux, 54280 Seichamps
}

\begin{abstract}
Résumé
Au cours des dernières années, de multiples méthodes visant à estimer la précision des mesures de superficie par comptage de points sont apparues; la diversité apparente de ces méthodes rend nécessaire une mise au point : elles sont équivalentes en première approximation, mais seule la méthode dérivée de la théorie des variables régionalisées est utilisable pour des études approfondies.
\end{abstract}

\section{1. - Rappel de la méthode}

On cherche à évaluer la surface d'une figure plane ; pour cela, on projette au hasard sur cette surface une grille de points ; cette grille de points, le plus souvent à maillage carré, peut être matérialisée par une feuille de papier millimétré transparent par exemple ; on comple le nombre de points tombant à l'intérieur de la figure ; on en déduit la surface qu'on cherche.

\section{2. - Rappel des différentes méthodes d'estimation de la précision} fig. 1) :

On distingue subjectivement quatre grands types de forme de surface (voir

- le type 1 : surface régulière

- le type 2 : surface irrégulière

- le type 3 : surface très irrégulière

- le type 4 : surface morcelée.

Les différents auteurs donnent des formules permettant de calculer les erreurs d'estimation; mais, ces formules fournissent soit une variance, soit un écart-type, soit un coefficient de variation ; il est par conséquent difficile de les comparer directe- 
ment ; elles ont donc été modifiées: la valeur $E$ qui est indiquée est l'erreur au seuil de 0,05 , exprimée en p. 100.

$$
E=196 \frac{\sigma}{S}
$$

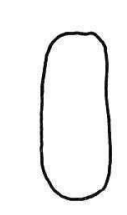

type 1

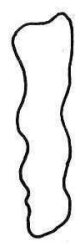

2

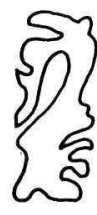

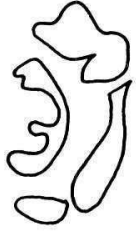

4 .

FIG. 1. - Quatre grands types de formes de surfaces.

Four types of area-forms.

\section{Définitions}

- $\mathrm{N}$ est le nombre de points tombant dans la surface

- $S$ est la surface à mesurer

- $P$ est son périmètre

- $N_{1}$ est un nombre dont l'évaluation est donnée dans Bouchon (1975) : c'est la mesure, en nombre de points, du double de la variation diamétrale dans l'une des deux directions perpendiculaires constituées par le maillage de points; si la figure n'a ni enclaves, ni golfes importants, c'est simplement le double du diamètre apparent dans cette direction; s'il y a des enclaves et des golfes, il faut y ajouter le double de leurs propres diamètres apparents (fig. 2).

$-\mathrm{N}_{2}$ est la variation diamétrale dans la direction perpendiculaire; on doit avoir :

$$
\mathrm{N}_{1} \geqslant \mathrm{~N}_{2}
$$

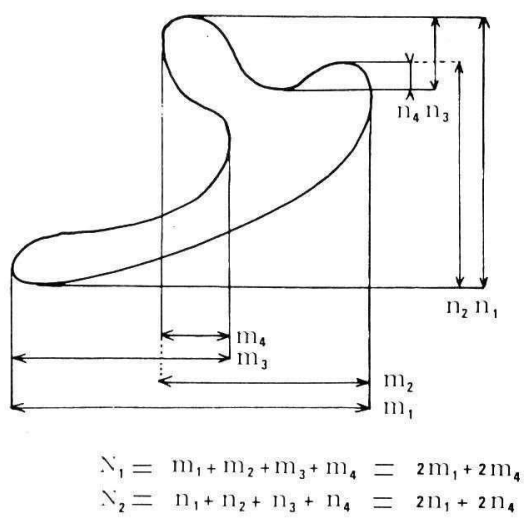

FIG. 2. - Variation diamétrale.

«Diamefrical variation ». 
Voici les formules proposées par les divers auteurs :

Bartet (1969) :

$$
E=100 N^{-2 / 3}
$$

Lœtsch (1973) :

$$
E=118,7 N^{-0,76}
$$

Bonnor (1975) :

$$
\begin{array}{ll}
E=71,78 N^{-0,64} & \text { (images de type 1) } \\
E=134,4 N^{-0,64} & \text { (images de type 2) } \\
E=153,1 N^{-0,58} & \text { (images de types } 3 \text { ou } 4)
\end{array}
$$

Zöhrer (1976)

$$
E=60,27 N^{-0,755}(P / \sqrt{S})^{0,457}
$$

Chevrou (1976) :

$$
\mathrm{E}=52,15 \mathrm{~N}^{-0,75}(\mathrm{P} / \sqrt{\mathrm{S}})^{0,5}
$$

Bouchon (1975), Matheron (1970) :

$$
E=196 N^{-1}\left(N_{2} / 12+0,0305 N_{1}^{2} / N_{2}\right)^{0,5}
$$

Les formules (1) à (6) ont été établies empiriquement ; les formules (7) et (8) sont basées sur des considérations théoriques.

Les deux premières formules ne tiennent pas compte de la forme de la figure mesurée ; les formules de Bonnor en tiennent compte, mais le choix du type de figure reste mal défini. Les formules (6) à (8) tiennent compte de la forme soit par l'intermédiaire du paramètre de forme $(P / \sqrt{S})$, soit par l'intermédiaire des nombres $N_{1}$ et $\mathrm{N}_{2}$ définis ci-dessus. Toutes ces formules ne donnent que des valeurs moyennes de l'erreur et ne sont utilisables que si la maille de la grille de points est petite par rapport à la figure, autrement dit si le nombre $\mathrm{N}$ de points est suffisamment grand. D'autre part Bonnor, Zöhrer et Chevrou mettent en garde contre un usage irréfléchi de ces formules, notamment dans les cas « aberrants» (Chevrou, 1976).

Remarque : Pour une forme circulaire, on a : $\mathrm{P} / \sqrt{\mathrm{S}}=2 \sqrt{\pi}$ et $\mathrm{N}_{1}=\mathrm{N}_{2}=4 \sqrt{\mathrm{N}} / \sqrt{\pi}$.

Les formules (6) à (8) deviennent alors :

$$
\begin{aligned}
& E=107,47 \mathrm{~N}^{-0,755} \\
& E=98,19 \mathrm{~N}^{-0,75} \\
& E=99,34 \mathrm{~N}^{-0,75} .
\end{aligned}
$$

On observe donc une très grande similitude entre ces formules, malgré leur apparente diversité ef leur origine théorique ou pragmatique.

Les quatre images de la figure 1 (réduites au $1 / 10$ sur la figure) ont une surface planimétrée de $100 \mathrm{~cm}^{2}$; leurs périmètres, mesurés au curvimètre, permettent de calculer les paramètres de forme qui sont respectivement de 4,09-5,2-9,85 ef 11,19. Pour le cercle on aurait $2 \sqrt{\pi}=3,54$; pour le carré ce paramètre est égal à 4 . 


\section{3. - Comparaison des méthodes}

Pour mesurer les surfaces on a utilisé 6 grilles différentes :

$$
5 \times 5 \mathrm{~cm} / 3 \times 3 / 2 \times 2 / 1 \times 1 / 0,5 \times 0,5 / \text { et enfin } 0,3 \times 0,3 \mathrm{~cm}
$$

Pour chacune de ces grilles et chacune des surfaces on a procédé à 10 évaluations de la superficie par complage de points. On a pu ainsi établir pour chacun des quatre types d'image la relation statistique entre la grandeur de la maille et l'erreur à craindre.

Parallèlement, et pour chacune des six méthodes, on a calculé l'erreur à l'aide des formules (1) à (8) ci-dessus.

TABLEAU 1

Colonne (1) : type d'image (fig. 1) ; colonne (2) : grandeur de la maille carrée ; colonne (3) : mesure directe de l'erreur ; colonne (4) à (9) : estimations par les 6 méthodes

Column (1) : type of form (fig. 1 : scale $1 / 10$ ) ; Column (2) : dod grid mesh in $\mathrm{cm}$;

Column (3) : direct measurement of the error in percentage ; Columns (4) to (9) : estimations by the 6 methods

\begin{tabular}{|c|c|c|c|c|c|c|c|c|}
\hline \multirow{2}{*}{$\begin{array}{c}\text { Image } \\
\text { (1) }\end{array}$} & \multirow{2}{*}{$\begin{array}{c}\text { Maille } \\
\text { (en cm) } \\
(2)\end{array}$} & \multirow{2}{*}{$\begin{array}{c}\mathrm{E}_{\mathrm{i}} \text { : } \\
\text { Erreur } \\
\text { mesurée } \\
\text { (p. 100) } \\
(3)\end{array}$} & \multicolumn{6}{|c|}{$\widehat{E_{i}}:$ Erreurs annoncées par les différentes méthodes } \\
\hline & & & $\begin{array}{c}\text { Bartet } \\
\text { (4) }\end{array}$ & $\begin{array}{l}\text { Lœtsch } \\
\text { (5) }\end{array}$ & $\begin{array}{c}\text { Bonnor } \\
(6)\end{array}$ & $\begin{array}{c}\text { Zöhrer } \\
\text { (7) }\end{array}$ & $\begin{array}{c}\text { Chevrou } \\
\text { (8) }\end{array}$ & $\begin{array}{c}\text { Bouchor } \\
\text { (9) }\end{array}$ \\
\hline Type 1 & $\begin{array}{c}5 \times 5 \\
3 \times 3 \\
2 \times 2 \\
1 \times 1 \\
0,5 \times 0,5 \\
0,3 \times 0,3\end{array}$ & $\begin{array}{r}42,52 \\
19,76 \\
10,76 \\
3,80 \\
1,34 \\
0,62\end{array}$ & $\begin{array}{r}39,69 \\
20,08 \\
11,70 \\
4,64 \\
1,84 \\
0,93\end{array}$ & $\begin{array}{r}41,39 \\
19,04 \\
10,28 \\
3,58 \\
1,25 \\
0,58\end{array}$ & $\begin{array}{r}29,56 \\
15,37 \\
9,15 \\
3,77 \\
1,55 \\
0,81\end{array}$ & $\begin{array}{r}40,28 \\
18,63 \\
10,10 \\
3,55 \\
1,24 \\
0,58\end{array}$ & $\begin{array}{r}37,29 \\
17,33 \\
9,43 \\
3,34 \\
1,18 \\
0,55\end{array}$ & $\begin{array}{r}38,37 \\
18,02 \\
9,89 \\
3,50 \\
1,24 \\
0,57\end{array}$ \\
\hline Type 2 & $\begin{array}{c}5 \times 5 \\
3 \times 3 \\
2 \times 2 \\
1 \times 1 \\
0,5 \times 0,5 \\
0,3 \times 0,3\end{array}$ & $\begin{array}{r}61,98 \\
28,81 \\
15,68 \\
5,54 \\
1,96 \\
0,91\end{array}$ & $\begin{array}{r}39,69 \\
20,08 \\
11,70 \\
4,64 \\
1,84 \\
0,93\end{array}$ & $\begin{array}{r}41,39 \\
19,04 \\
10,28 \\
3,58 \\
1,25 \\
0,58\end{array}$ & $\begin{array}{r}55,35 \\
28,78 \\
17,13 \\
7,05 \\
2,90 \\
1,51\end{array}$ & $\begin{array}{r}44,95 \\
20,79 \\
11,27 \\
3,96 \\
1,39 \\
0,64\end{array}$ & $\begin{array}{r}42,04 \\
19,54 \\
10,64 \\
3,76 \\
1,33 \\
0,62\end{array}$ & $\begin{array}{r}47,08 \\
20,93 \\
11,37 \\
4,02 \\
1,42 \\
0,66\end{array}$ \\
\hline Type 3 & $\begin{aligned} 5 & \times 5 \\
3 & \times 3 \\
2 & \times 2 \\
1 & \times 1 \\
0,5 & \times 0,5 \\
0,3 & \times 0,3\end{aligned}$ & $\begin{array}{r}82,69 \\
38,43 \\
20,92 \\
7,40 \\
2,61 \\
1,22\end{array}$ & $\begin{array}{r}39,69 \\
20,08 \\
11,70 \\
4,64 \\
1,84 \\
0,93\end{array}$ & $\begin{array}{r}41,39 \\
19,04 \\
10,28 \\
3,58 \\
1,25 \\
0,58\end{array}$ & $\begin{array}{r}68,51 \\
37,88 \\
23,67 \\
10,59 \\
4,74 \\
2,62\end{array}$ & $\begin{array}{r}60,19 \\
27,83 \\
15,09 \\
5,30 \\
1,86 \\
0,86\end{array}$ & $\begin{array}{r}57,87 \\
26,90 \\
14,64 \\
5,18 \\
1,83 \\
0,85\end{array}$ & $\begin{array}{r}59,68 \\
27,69 \\
15,09 \\
5,33 \\
1,88 \\
0,88\end{array}$ \\
\hline Type 4 & $\begin{aligned} 5 & \times 5 \\
3 & \times 3 \\
2 & \times 2 \\
1 & \times 1 \\
0,5 & \times 0,5 \\
0,3 & \times 0,3\end{aligned}$ & $\begin{array}{r}75,86 \\
35,26 \\
19,19 \\
6,78 \\
2,40 \\
1,11\end{array}$ & $\begin{array}{r}39,69 \\
20,08 \\
11,70 \\
4,64 \\
1,84 \\
0,93\end{array}$ & $\begin{array}{r}41,39 \\
19,04 \\
10,28 \\
3,58 \\
1,25 \\
0,58\end{array}$ & $\begin{array}{r}68,51 \\
37,88 \\
23,67 \\
10,59 \\
4,74 \\
2,62\end{array}$ & $\begin{array}{r}63,81 \\
29,51 \\
15,99 \\
5,62 \\
1,97 \\
0,91\end{array}$ & $\begin{array}{r}61,68 \\
28,67 \\
15,60 \\
5,52 \\
1,95 \\
0,91\end{array}$ & $\begin{array}{r}62,58 \\
29,09 \\
15,83 \\
5,60 \\
1,98 \\
0,92\end{array}$ \\
\hline
\end{tabular}




\section{Résultats et première conclusion}

Le tableau 1 donne les résultats des comparaisons. D'une manière générale les différentes méthodes fournissent des résultats corrects pour les formes simples (type I) et, mise à part la méthode BONNOR, sous-estiment l'erreur dans les autres cas. Un test fait sur l'écart relatif quadratique moyen $Q=\sqrt{\frac{\sum\left[\left(E_{i}-\hat{E}_{i}\right) / E_{i}\right]^{2}}{24}}$
permet de classer les 6 méthodes:

$\begin{array}{ll}\mathrm{Q} \text { (Bouchon, Matheron) } & =0,217 \\ \mathrm{Q} \text { (Zöhrer) } & =0,220 \\ \mathrm{Q} \text { (Chevrou) } & =0,246 \\ \mathrm{Q} \text { (Bartet) } & =0,322 \\ \mathrm{Q} \text { (Løtsch) } & =0,389 \\ \mathrm{Q} \text { (Bonnor) } & =0,518 .\end{array}$

Les trois premières donnent des résultats comparables, mais il est nécessaire de mesurer le périmètre pour les méthodes Zöhrer et Chevrou si on veut atteindre cette précision ; ceci retire une grande partie de leur intérêt à ces deux méthodes puisqu'alors il est aussi simple de planimétrer la surface à mesurer.

Pour une estimation rapide, la méthode Bartet peut être suffisante ; pour des estimations plus précises la méthode Bouchon-Matheron est la plus recommandable.

\section{4. - « Zitterbewegung » (terme fluctuant)}

Les formules présentées au paragraphe 2 ne donnent que des valeurs moyennes de l'erreur. En effet, dans la réalité, si la surface à mesurer est repérée dans un système d'axes orthogonaux et si le maillage, qu'on supposera ici carré et de maille a, fait un angle $t$ avec ces axes, on peut montrer que l'erreur $E$ est non seulement fonction de $\mathrm{N}$ et du coefficient de forme $\mathrm{P} / \sqrt{\mathrm{S}}$, mais aussi de a et $t$.

$$
E=E(N, P / \sqrt{S}, a, t) \text {. }
$$

Les formules (1) à (7) ci-dessus correspondent à la moyenne qu'on obtiendrait en faisant varier a de 0 à une valeur $A$ grande par rapport à la figure ef $t$ de 0 à $\pi / 4$ :

$$
E=\frac{4}{\pi \mathrm{A}} \iint E(N, P / \sqrt{S}, a, t) d a d t .
$$

La formule (8), par l'intermédiaire des nombres $N_{1}$ et $N_{2}$ fournit l'erreur en fonction de l'inclinaison $\dagger$ du maillage et n'est donc que la moyenne par rapport aux grandeurs de mailles; dans le cas du rectangle on peut calculer directement la moyenne de $E$ relativement à la maille; la figure 3 indique par exemple quelles sont les valeurs de $t$ pour lesquelles l'erreur moyenne sera la plus faible : pour des rectangles allongés $(b / B<0,55)$ la variance minimale est obtenue pour un angle de $50 \mathrm{gr}$. Pour des rectangles peu allongés $(b / B>0,68)$ la variance minimale est obtenue lorsque le maillage est parallèle aux côtés du rectangle. 
Une étude analogue faite sur l'ellipse, montre que la variance minimale est toujours obtenue pour un angle de $50 \mathrm{gr}$ entre le maillage et les axes de l'ellipse.

Dans la réalité l'erreur dépend du maillage ; la figure 3 indique par exemple qu'en moyenne pour un carré $(b / B=1)$ la variance est minimale lorsque le maillage est parallèle aux côtés.

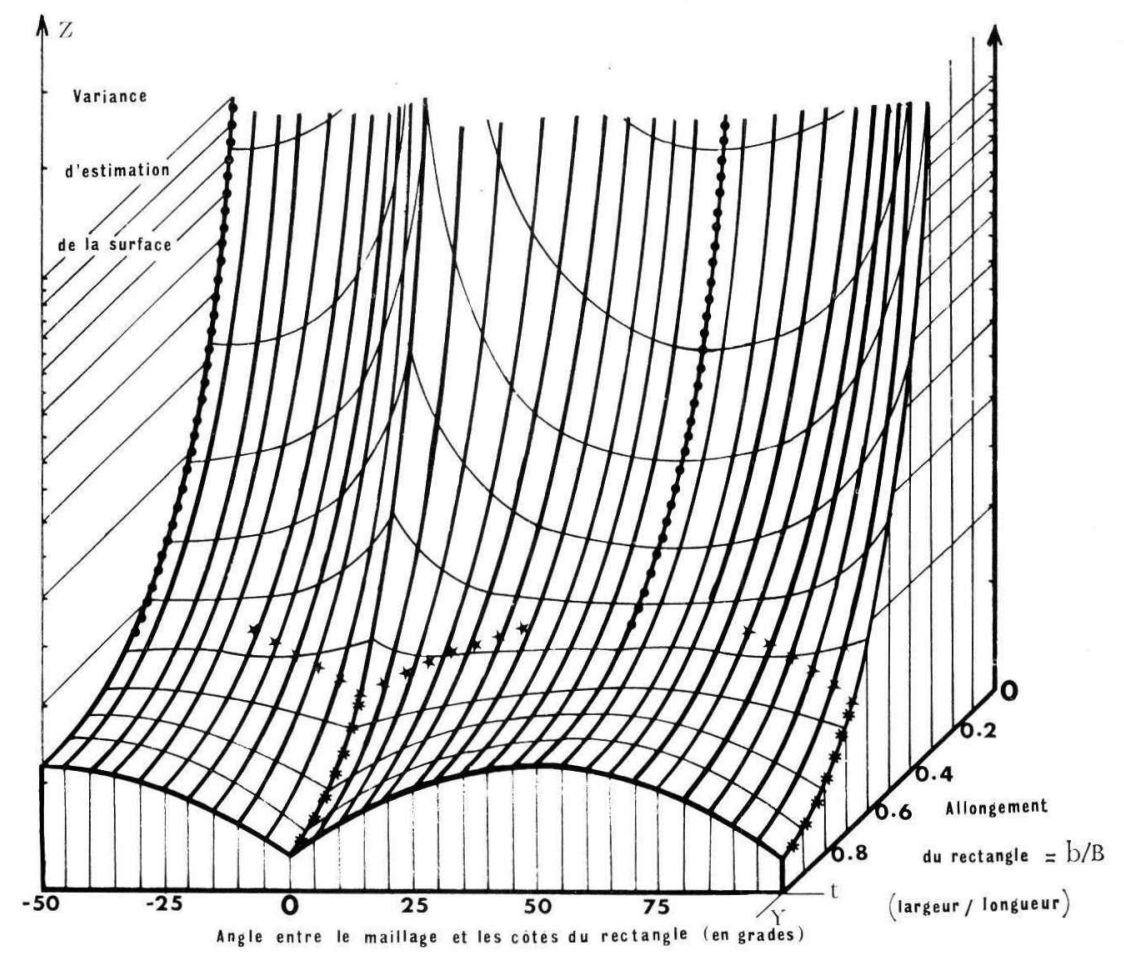

FIG. 3. - Variance d'estimation de la surface d'un rectangle pour des rapports largeur/longueur variant de 0 à 1 et une inclinaison du maillage par rapport aux côtés du rectangle variant de 0 à $50 \mathrm{gr}$.

Variance of the measurements of a rectangular area (axis Z) in terms of axis $t$ : angle between the sides of the rectangle and the directions of the dot-grid; axis $y:$ length/width ratio.

For $0,68 \leqslant b / B \leqslant 1$, the minimum of the variance is obtained for $t=0 \mathrm{gr}$ (or $100 \mathrm{gr}$ ). For $b / B \leqslant 0,55$, the minimum is obtained for $t= \pm 50 \mathrm{gr}$. But we have here only means; actually the real variances depend also on the width of the mesh of the dod grid (see § 4).

Cependant si l'on veut mesurer la surface d'un carré de $2,5 \mathrm{~cm}$ de côté à l'aide d'un maillage de $1 \mathrm{~cm}$ de côté, le calcul direct montre que la variance est égale à $3,19 \mathrm{~cm}^{4}$ lorsque $t=0$ et seulement $1,62 \mathrm{~cm}^{4}$ lorsque $\dagger=50 \mathrm{gr}$. Il est donc nécessaire de distinguer la moyenne fournie par les formules, du risque lié à une certaine incongruité entre les dimensions du maillage et celles de la figure. On étudiera d'abord les mesures de longueurs à l'aide de graduations linéaires ; les résultats seront appliqués au cas des mesures de surfaces rectangulaires puis au cas général. 


\section{1. - Mesure de longueur}

Que se passerait-il si on tentait de mesurer une longueur b en lui appliquant au hasard une graduation linéaire de maille $a$ ? (par exemple pour un double décimètre gradué en $\mathrm{mm}, \mathrm{a}=1 \mathrm{~mm}$ ).

Supposons que l'on ait :

$$
b=m a+r
$$

$m$ étant un nombre entier

et $r$ le reste de la division de b par $a$.

En appliquant la graduation a au hasard sur b, on trouvera donc comme longueur : ma avec la probabilité $(a-r) / a$ et $(m+1)$ a avec la probabilité $r / a$; ce qui donne bien comme moyenne : $m a+r=b$.

La variance de ces mesures sera :

$$
\begin{aligned}
& \sigma^{2}=[m a-(m a+r)]^{2}[(a-r) / a]+[(m+1) a-(m a+r)]^{2} r / a \\
& \sigma^{2}=r(a-r) .
\end{aligned}
$$

On observe donc que la variance est nulle lorsque la longueur à mesurer est un multiple exact de la graduation $(r=0)$ et que cette variance est maximale quand $r=a / 2 *$.

Pour une valeur de a donnée, la variance a une valeur moyenne : $\bar{\sigma}^{2}=a^{2} / 6$. Mais, en réalité, on ne connaît pas b, et tout ce qu'on peut affirmer c'est que la variance sera comprise entre zéro et $a^{2} / 4$. C'est cette fluctuation imprévisible (puisque $b$ est inconnue) autour de la moyenne $a^{2} / 6$ qu'on appelle «Zitterbewegung » ou « terme fluctuant $\gg$.

\section{2. - Cas du rectangle}

Supposons que la longueur B du rectangle soit grande par rapport à sa largeur b ; on applique une grille de points parallèlement aux grands côtés, ces parallèles étant distantes de a les unes des autres ; on peut montrer (Chevrou, 1976) que, comme dans le cas précédent, l'erreur est liée au reste $r$ de la division de b par a :

$$
\mathrm{b}=\mathrm{ma}+\mathrm{r} .
$$

Dans les exemples présentés ci-dessous le maillage utilisé sera toujours carré et de maille $a=1 \mathrm{~cm}$.

\section{Exemple 1 :}

Rectangle $B=20 \mathrm{~cm}, b=2,5 \mathrm{~cm}$.

Si le maillage est parallèle aux côtés du rectangle, un comptage sur deux sera égal à $40\left(40 \mathrm{~cm}^{2}\right)$ et l'autre à $60\left(60 \mathrm{~cm}^{2}\right)$ (voir fig. 4).

\footnotetext{
* La distribution des erreurs dans ce cas est très particulière : une mesure sur deux est égale à $b-a / 2$, efl'autre $a ̀ b+a / 2$. La variance ainsi calculée conduit par conséquent à une mesure assez grossière de l'erreur à craindre.
} 


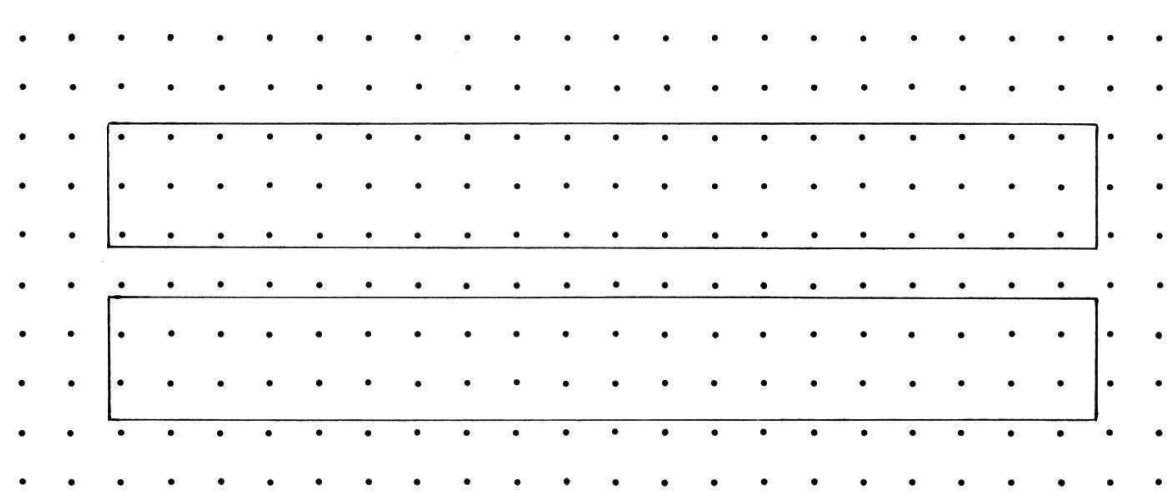

FIG. 4. - Surface d'un rectangle de côtés $(B=20 \mathrm{~cm}) \times(b=2,5 \mathrm{~cm})$ mesuré à l'aide d'une grille de $1 \mathrm{~cm}$ parallèle aux côtés du rectangle (échelle $1 / 2$ ). Une fois sur deux on obtient $40 \mathrm{~cm}^{2}$ et une fois sur deux $60 \mathrm{~cm}^{2}$.

Area of a rectangle : $B=20 \mathrm{~cm} ; b=2,5 \mathrm{~cm}$. The dot grid mesh measures $1 \mathrm{~cm}($ scale $1 / 2)$. Here the angle between the grid and the sides is zero. The measurement gives or $40 \mathrm{~cm}^{2}$ or $60 \mathrm{~cm}^{2}$.

\section{Exemple 2 :}

Rectangle $B=20 \mathrm{~cm} ; b=5 \mathrm{~cm}$.

Si le maillage est parallèle aux côtés, on aura exactement 100 points quelle que soit la position du maillage : l'erreur sera constamment nulle. Mais si le maillage est à $50 \mathrm{gr}$, alors les lignes de points seront distantes de $a(50)=\sqrt{2} / 2=0,7071 \mathrm{~cm}$. Le reste de la division de $\mathrm{b}=5 \mathrm{~cm}$ par $\mathrm{a}(50)=0,7071$ n'est pas nul et c'est pour cette inclinaison qu'on a une erreur maximale.

\section{Exemple 3 :}

$B=20 \mathrm{~cm} ; b=4,98 \mathrm{~cm}$. Ce cas paraît très proche du précédent ; pourtant c'est pour une inclinaison du maillage de $29,52 \mathrm{gr}$ que la distance entre les lignes, qui est de a $(29,52)=0,4472 \mathrm{~cm}$, conduit à une erreur maximale.

Lorsqu'on fait des mesures de superficie par comptage de points on ne connaît rien de la figure à mesurer ; les exemples précédents montrent donc qu'il est impossible de prévoir la grandeur de la maille a et l'inclinaison du maillage minimisant l'erreur. Tout ce qu'on peut dire c'est que pour une maille a donnée, les risques les plus grands sont obtenus pour les distances entre lignes de points successives les plus grandes. On montre que ces distances les plus grandes correspondent aux valeurs de $\operatorname{tg} \dagger$ qui sont égales au rapport de deux nombres entiers : $\operatorname{tg} \dagger=k / j$.

La distance entre deux lignes de points contiguës est alors $a / \sqrt{k^{2}}+j^{2}$. Les risques les plus grands sont donc à attendre pour les coupes $(k, j)$ suivants : $(0,1),(1,0),(1,1)$, $(1,2),(2,1)$ etc... d'où les valeurs de t qu'il faut éviter :

$$
0 \mathrm{gr}, 100 \mathrm{gr}, 50 \mathrm{gr}, 29,52 \mathrm{gr}, 70,48 \mathrm{gr} \text {, etc... }
$$

\section{3. - Cas général}

Le phénomène de «Zitterbewegung 》 («terme fluctuant») démontré pour les mesures de longueur $(\$ 4.1)$ et de superficies de rectangles $(\S 4.2)$ est très général ; l'exemple du carré présenté au début du paragraphe 4 s'explique facilement : lorsque les côtés de longueur $b=2,5 \mathrm{~cm}$ sont parallèles aux mailles de longueur $a=1 \mathrm{~cm}$, 
le reste de la division de b par a étant égal à $a / 2$ conduit à une variance maximale. Matheron (1970) fournit la courbe obtenue pour le cercle; le tableau 2 donne pour un cercle de $100 \mathrm{~cm}^{2}$, l'erreur de mesure obtenue avec des maillages carrés de différentes dimensions : ce tableau montre l'ampleur du terme fluctuant et combien les formules (1) à (8) sont approximatives puisqu'elles ne dépendent que du nombre de points compté et pour certaines d'entre elles de la forme ; ici par exemple on voit que l'erreur est pratiquement la même pour des mailles de $5 \mathrm{~cm}$ et de $2,83 \mathrm{~cm}$ alors que les nombres de points comptés sont en moyenne respectivement de 4 et de 12,5.

\section{TABLEAU 2}

Erreur en fonction de la grandeur de la maille dans la mesure de la surface d'un cercle de $100 \mathrm{~cm}^{2}$.

Measurement of the area of a $100 \mathrm{~cm}^{2}$ circle.

The error depends on the dimension of the dod grid mesh : $a$. For $a=2,83 \mathrm{~cm}$ (about 12 points in the circle) the error is a little bigger than for $a=5 \mathrm{~cm}$ (only 4 points)

\begin{tabular}{ccc}
\hline $\begin{array}{c}\text { Dimension } \\
\text { de la maille } \\
a\end{array}$ & $\begin{array}{c}\text { Nombre moyen } \\
\text { de points comptés } \\
N\end{array}$ & $\begin{array}{c}\text { Erreur } \\
196 \sigma / \mathrm{s}\end{array}$ \\
\hline $5 \mathrm{~cm}$ & 4 & $19,15 \mathrm{p} .100$ \\
$4,26 \mathrm{~cm}$ & 5,5 & $34,79 \mathrm{p} .100$ \\
$4 \mathrm{~cm}$ & 6,25 & $29,15 \mathrm{p} .100$ \\
$2,83 \mathrm{~cm}$ & 12,5 & $19,40 \mathrm{p} .100$ \\
\hline
\end{tabular}

\section{Deuxième conclusion}

Dans le cas de formes géométriques simples on peut calculer le terme fluctuant ; mais dans le cas général sa variation en fonction de la grandeur de la maille et de l'inclinaison est imprévisible ; les seules indications pratiques qu'on peut donner sont les suivantes:

- Lorsque l'image à mesurer est allongée, il faut éviter que les alignements de points de la grille soient parallèles au plus grand diamètre de l'image.

- En moyenne, mais en moyenne seulement, plus la maille est petite, meilleure est la précision.

\section{Remarque finale}

Les formules présentées au paragraphe 2, ainsi d'ailleurs que la comparaison des méthodes du paragraphe 3, font l'hypothèse implicite qu'un certain nombre de réalisations de comptages de points permettent d'atteindre une estimation directe de l'erreur $E(N, P \sqrt{S})$; or les « erreurs mesurées » qui figurent en troisième colonne du tableau 1 , bien qu'obtenues par régression, dépendent encore en réalité des grandeurs des mailles figurant en deuxième colonne, et en toute rigueur il n'est pas possible d'utiliser des mesures de $E(N, P \sqrt{S}, a)$ pour tester la validité de formule qui ne donnent que $E(N, P \sqrt{S})$. 


\section{Summary}

Measurement of areas by dot grids : comparison of 6 methods

For some years, a number of methods have been developed to measure area with the help of dot grids. Estimates of the accuracy shown by these methods are compared; all are equivalent for a quick estimation ; for further investigations only the method derived from the theory of regionalized variables is suitable.

\section{Références bibliographiques}

BARTET J. H. et GRAND J. P., 1969. Mesures de surfaces par comptage de points. La Forêt, oct. 1969, PP. 8-14.

BONNOR G. M., 1975. The error of area estimates from dot grids. Can. J. For. Res., 5, 10 (1975), 10-17.

BOUCHON J., 1975. Précisions des mesures de superficie par comptage de points. Ann. Sci. Forest., 1975, $32(2), 131-134$.

CHEVROU R. B., 1976. Précisions des mesures de superficie estimée par grille de points ou intersections de parallèles. Ann. Sci. Forest., 1976, 33 (4), 257-270.

LOETSCH F. et al., 1973. Forest Inventory. BLV München, Tome II, pp. 33 à 35.

MATHERON G., 1970. La théorie des variables régionalisées. Les Cahiers du Centre de Morphologie Mathématique, Fascicule 5, E.N.S.M.P., Fontainebleau, 1970, 212 p.

ZÖHRER F., 1976. Die Genauigkeit des Ermittlung von Waldflächen durch systematische Punktstichproben I (Einzelflächen). Forstwissenschaftliches Centralblatt, 1976, 95 (5/6), 294-301. 